\title{
Admission control in wireless ad hoc networks: a survey
}

\author{
Lyes Khoukhi" ${ }^{*}$, Hakim Badis ${ }^{2}$, Leila Merghem-Boulahia ${ }^{1}$ and Moez Esseghir ${ }^{1}$
}

\begin{abstract}
Wireless mobile ad hoc networks (MANETs) have emerged as a key technology for next-generation wireless networking. Because of their advantages over other wireless networks, MANETs are undergoing rapid progress and inspiring numerous applications. However, many technical issues are still facing the deployment of this technology, and one of the most challenging aspects is the quality of service (QoS) provisioning for multimedia real-time applications. MANETs are expected to offer a diverse range of services to support real-time traffic and conventional data in an integrated fashion. Because of the diversified QoS requirements of these services, QoS models are needed for an efficient usage of network resources. One of the most crucial mechanisms for providing QoS support is admission control (AC). AC has the task of estimating the state of network's resources and thereby to decide which application data flows can be admitted without promising more resources than are available and thus violating previously made guarantees. In order to provide a better understanding of the AC research challenges in MANETs, this paper presents a detailed investigation of current state-of-the-art AC models in ad hoc networks. A brief outline of the admission function, feedback to route failures, as well as the advantages and drawbacks of each discussed model are given.
\end{abstract}

Keywords: Quality of service, Wireless mobile ad hoc networks, Admission control

\section{Review} Introduction

Mobile ad hoc networks (MANETs) offer unique advantages and flexibility for certain environments and strategic applications. They are constructed by a set of mobile nodes which are autonomously connected via multihop wireless communications (Figure 1). Without any preconfigured and centrally controlled network infrastructure, they can be created and used 'anytime, anywhere.' In fact, since all nodes are allowed to be mobile, the composition of such networks is necessarily timevarying. Not relying on complex and expensive infrastructure, which is required by their traditional counterparts, MANETs can operate in several environments where conventional networks fail [1,2]. Such perceived benefits elicited urgent attention in the early days among military and rescue agencies in the use of ad hoc networks, especially under disorganized or hostile environments. It is

\footnotetext{
* Correspondence: lyes.khoukhi@utt.fr

'Autonomic Networking Environment, CNRS UMR-STMR 6279, University of Technology of Troyes, Troyes 10000, France

Full list of author information is available at the end of the article
}

vital to note that one of the major factors in the growing interest in MANETs was the improving capacities and ubiquitous nature of mobile devices, as well as the development of the unifying IEEE 802.11 standard [3] for wireless networking. Most laptop computers and many personal digital assistants now come with 802.11compliant air interfaces. With the option to operate them in ad hoc mode, 802.11 is the primary enabling technology of MANETs [4-6].

Providing quality of service (QoS) to users in a MANET is a key concern for service providers. Many suggested applications consist of real-time voice and video traffic that require QoS support for effective communication. The purpose of any QoS support model is to offer services with guarantees in terms of delay, bandwidth, jitter, or packet loss. To provide such guarantees in dynamic ad hoc networks, the media access control (MAC) layer is responsible for bandwidth allocation at individual devices, while the network layer must consider resources along the whole path of transmission [7]. One of the most crucial mechanisms for providing QoS guarantees is admission control (AC). AC aims to estimate the state of network's 


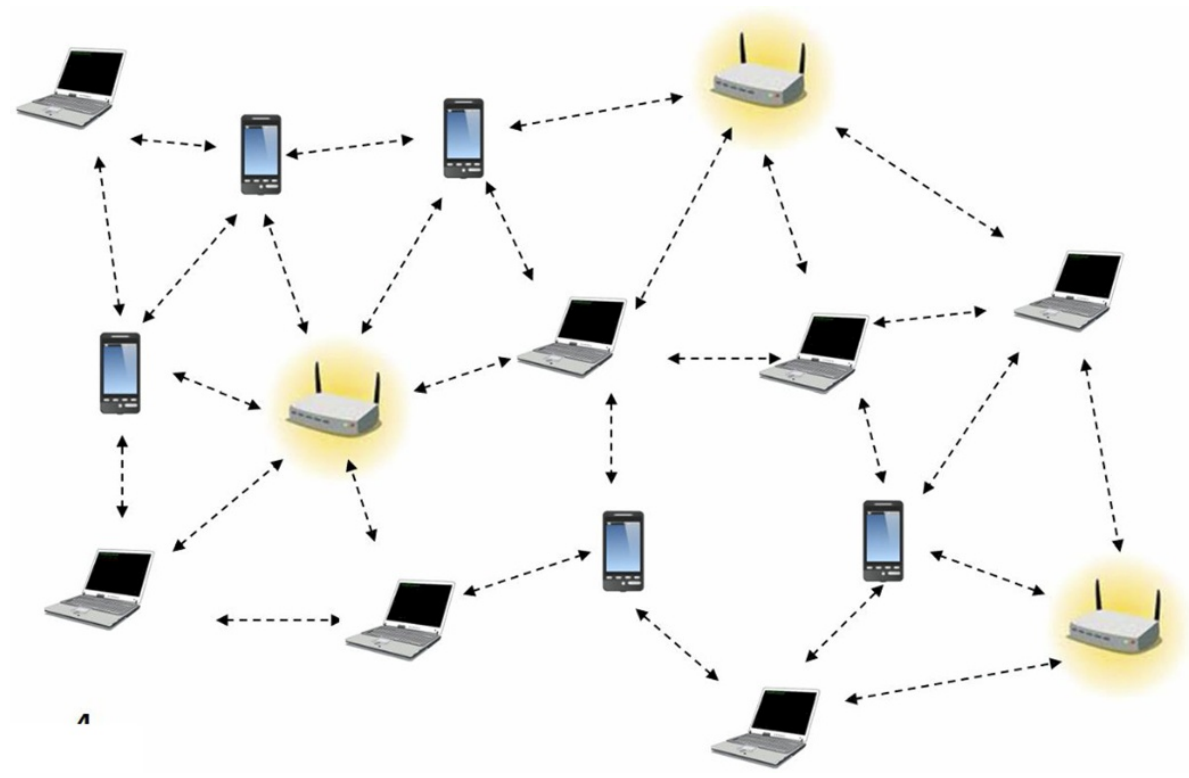

Figure 1 Example of MANET.

resources and thereby to decide which application data flows can be admitted without promising more resources than are available and thus violating previously made guarantees. AC has the task of controlling the usage and allocation of network resources for various applications requiring additional services. $\mathrm{AC}$ is a key component in multimedia systems, which needs to allow the bandwidth to be used by flows only when it is available.

In order to provide a better understanding of the $\mathrm{AC}$ research challenges in MANETs, this paper presents a detailed investigation of current works regarding $\mathrm{AC}$ models for ad hoc networks. An outline of the admission function, feedback to route failures, as well as the advantages and drawbacks of each AC model presented in this paper are given. The rest of the paper is organized as follows: the 'Design challenges of AC models in MANETs' section aims to provide an overview of some important issues regarding the design of AC models in ad hoc networks. The 'AC models in ad hoc networks' section lists the relevant admission control models found in the literature, illustrates their classification according to the method adopted for this survey, and describes their operations, advantages, and drawbacks. Finally, the 'Conclusions' section presents some concluding remarks, summarizes the trends in the field, and highlights potential areas of future work.

\section{Design challenges of AC models in MANETs}

Due to the probabilistic nature of wireless medium, admission control for ad hoc networks has many challenging problems to solve. The characteristics of the shared wireless medium do not provide a unified view of the medium to all nodes due to the physical differences between wired and wireless communication. The assumptions made by some researchers in the design of QoS AC models are first, as for resource availability, each wireless mobile device is assumed to be able to monitor (implicitly or explicitly) the available resources on each of its outgoing links. Second, as for resource reservation, a medium access protocol is supposed to be able to resolve media contention and support resource reservation at the MAC layer. Before presenting some remarkable AC models proposed in the literature, we first discuss some challenges facing the design of such models. Note that many of the discussed challenges are the same as those posed to QoS-aware protocols, which were discussed in the literature. Some critical issues to consider in the design of $\mathrm{AC}$ models are the following:

- Node mobility: the mobile devices in MANETs may move randomly and independently. This means that the topology information has a limited lifetime and must be updated frequently to allow data packets to be routed to their destinations. Furthermore, the dynamic topology can also lead to violations of QoS assurance without breaking routes because a transmitting node may move into the sensing range of another transmitter, thereby increasing its interference and reducing its channel access time $[8,9]$.

- Channel contention: even if the MAC protocol in use is not the single-channel 802.11 scheme, mobile devices in ad hoc networks should communicate on a common channel. However, this leads to channel 
contention and interference problems, which can impact on the fraction of channel capacity available to a mobile device. Another consequence of channel contention is mutual contention and interference between nodes on a route forwarding the packets of a data session [8].

- Unreliable wireless channel: received signals are prone to bit errors due to interference from other transmissions, thermal noise, shadowing, and multipath fading effects. Such errors may lead to increased packet delays and possible congestion, causing more packets to be dropped [10].

- Connectivity issue: a mobile device may lose connectivity with the rest of the group just because it has wandered off too far or its power reserve has dropped under a certain threshold. A session that was admitted based on the available route may be starved of transmission opportunities if some nodes lose connectivity with others. The session would then need to be re-admitted on a new route.

- Lower algorithmic complexity: one main design criterion of AC models is related to lower algorithmic complexity to facilitate limited-bandwidth and lowpower QoS solutions that can be embedded into lowcost mobile devices' microprocessors and to extend the lifetime of the network without endangering efficient and reliable communications between mobile nodes $[7,9]$. Usually, this is impacted by the kind of traffic (e.g., CBR voice real-time traffic, rt-VBR video conferencing flows) generated by the source devices, available buffer sizes within intermediate nodes, nodes' connectivity, QoS constraints, etc. In a wireless ad hoc environment, this is further impacted by the fact that the common medium is the wireless channel; this means that control and data packets can get corrupted at physical layer (due to errors and interferences) or at MAC layer (due to collision), etc. Wireless links between mobile devices are 'dynamic' in that they come and go over time, i.e., two nodes which could speak to each other suddenly cannot, and vice versa [7]. Furthermore, mobile devices usually suffer from limited resources in terms of bandwidth, processing and memory capacities, and energy.

\section{AC models in ad hoc networks}

The allocation of network resources is always necessary for communication over a shared medium in a multihop wireless network; this demands a very different perspective on network QoS admission control management. In this section, we describe some current research in the area classified into two categories: single-hop AC (characterized by soft constraints) and multihop AC (characterized by hard constraints). Because of the simplicity of the single-hop wireless environment, we focus our survey mainly on multihop $A C$, where several considerations have been considered, e.g., network information updating, interaction-based routing, and QoS strategy (Figure 2). Where the AC models are named, we use the names given by the proposing papers; otherwise, the AC models are named based on their reference number in this paper.

\section{Single-hop AC}

This section describes briefly some of the main AC models proposed for the single-hop wireless environment, which refers to the wireless infrastructure mode. These AC models, proposed by some researchers, respond to the limitations of contention-based access control schemes targeted at delivering classic data services in IEEE 802.11. The IEEE 802.11e standard [11] defines the basic procedures for contention-based admission control for enhanced distributed channel access (EDCA). A mobile station which needs to start a new real-time session will first transmit an add-traffic-stream request (ADDTS.request) frame to the QoS access point (QAP). The station indicates the traffic parameters such as the nominal MAC service data unit size, mean data rate, and surplus bandwidth allowance in the 'ADDTS.request' frame. Based on these parameters and the network state, the QAP makes an admission decision and sends back the results in the 'ADDTS.response' frame. Note that the network operators are free to implement their own algorithms of admission decision because the IEEE 802.11e

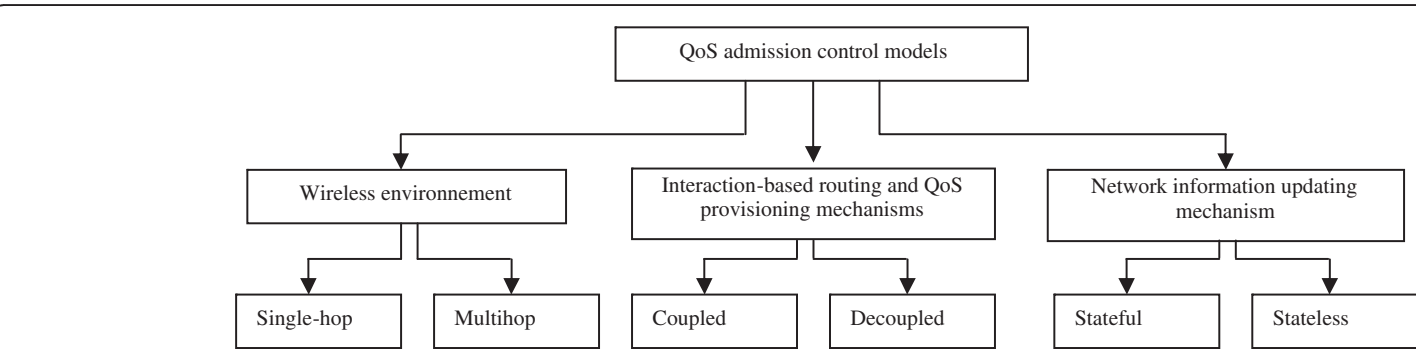

Figure 2 Classification of AC QoS models. 
only describes the signaling frame format for admission control [12].

Xiao and Li [13] proposed a distributed asynchronous cooperation (DAC) protocol. In DAC, the QAP announces the transmission budget via beacons, which is the additional amount of time available for each AC during the next beacon interval. Each station determines an internal transmission limit per $\mathrm{AC}$ for each beacon interval based on the successfully used transmission time during the previous beacon period and the transmission budget announced from the QAP. When the transmission budget for an AC is depleted, a new flow will not be able to obtain any transmission time, and existing flows will not be able to increase their transmission time too. The main weakness of the DAC model is that it can only protect existing flows when the traffic load is not heavy. In addition, this scheme does not provide a direct relationship between transmission opportunity (TXOP) parameters and QoS requirements related to user applications $[13,14]$.

The authors in [15] proposed a similar DAC-based scheme which includes two-level protection and guarantee mechanisms. The principle of the first level is to protect each existing voice and video flows from the new and other existing voice and video flows. As for the second level, it protects the existing QoS flows from the best-effort data traffic. When the number of active stations is large, the DAC-based scheme increases the initial contention window size and inter-frame space for best-effort data traffic. Because this two-level scheme is based on the DAC, it also has the problems of performance oscillation and lack of direct QoS relationship with applications.

In [16], Zhang and Zeadally have proposed HARMONICA model in which the access point dynamically selects the best channel access parameters for each traffic class to optimally match their QoS requirements. This protocol periodically samples the link-layer quality indicator parameters (drop rate, end-to-end delay and throughput) for each traffic class. Two adaptation algorithms over different time scales are employed to select the channel access parameters, which can best match the QoS requirements of each traffic class and the current channel contention level. The advantage of HARMONICA is that it offers the possibility to match the QoS needs and to guarantee a minimal bandwidth for best-effort traffic. However, the manner of finding the optimal increment or decrement in the value of channel access parameters remains the main limitation of this AC model.

Dennis and Tim [17] proposed an admission control algorithm for the 802.11e EDCA that considers the dynamic wireless network conditions such as the number of active sessions and the parameters adopted for these sessions. The authors argued that by predicting the achievable throughput of the data sessions and avoiding channel overloading, the QoS of existing sessions can be maintained. For that aim, they integrated some basic ideas of EDCA into their admission control algorithm and extend its capability to offer bandwidth guarantees, rather than providing a relative prioritized service. The algorithm deals with the parameters of transmission opportunity duration and minimum contention window size, and identifies the suitable values that should be used for different sessions. One of the advantages of this proposal is that it can predict the achievable throughput value for each session. This information may be a helpful guiding indication to efficiently supervise bandwidth provision in EDCA. However, as shown in simulations that the $\mathrm{CW}_{\text {min }}$ parameter affects both the delay and bandwidth, and when a larger TXOP is assigned to voice session, the delay jitter is increased.

In [18], Wu and Bertsekas considered the problem of optimal admission control in a single-hop wireless infrastructure mode to determine whether or not to accept a new session request, given a particular configuration of users of various classes in various regions. The authors assumed the existence of an algorithm that can determine, for any distribution of users of various classes in various regions, whether there is a feasible power assignment satisfying the signal-to-noise requirements for all users and, if so, provide a unique power assignment for the distribution. They formulated the problem as a Markov decision process to provide a technique that is enough to be applicable and can be implemented in real time in a distributed manner between the cells. One of the advantages of such technique is that it can incorporate an arbitrary amount of detail necessary to describe real wireless infrastructure systems. However, this work is based on a set of assumptions (like the existence of algorithm which assigns dynamically the resources to users, the probability of moving is known a priori, etc.) that may restrict the model in the implementation phase.

In [19], Abdrabou and Zhuang proposed a new approach to provide stochastic delay guarantees via a distributed model-based call admission control for IEEE 802.11 single-hop networks. The authors used a linklayer channel model to characterize the variations of the channel service process in a non-saturated case via a Markov-modulated Poisson process model (MMPP). The performance evaluation showed that the MMPP link-layer approach can be used successfully in allocating resources with stochastic delay guarantees. However, other parameters, such as throughput and packet loss, have not been considered in MMPP.

As discussed above, the single hop-based AC concept model does not pose big challenges, as opposite to the 
multihop network characterized by the lack of centralized control and the dynamically changing network topology, as it will be described in what follows.

\section{Multihop $A C$}

To protect the existing sessions and satisfy the QoS requirements of new flows in multihop ad hoc networks, several admission control schemes have been proposed. In the following sections of this paper, the protocol descriptions are grouped into sections based on the classification method in Figure 3.

Routing-decoupled AC schemes This sub-section deals with $\mathrm{AC}$ schemes that are decoupled from routing schemes, which means that a route for a requesting flow has been explored prior to testing its resources. In such schemes, the decision of admission control is achieved based on 'probing' of the route by previously admitted flows or special probe packets.

In [20], the authors proposed a probe-based call admission control scheme (ProbeCast) with QoS guarantees for inelastic flows (i.e., flows that cannot be dynamically adjusted to traffic and load condition). In ProbeCast, a path is probed for capacity availability. If an intermediate link along the probed path fails to meet the QoS requirement, the flow is 'pushed back' via backpressure upstream to an intermediate branch or possibly to the source. To achieve this, the probing scheme is associated with a distributed fairness scheme,
Neighborhood Proportional Drop, which enforces uniform drop probabilities among flows competing in the same contention domain. Each node estimates own packet drop probability and propagates this information by piggybacking to neighbors. The received flow has, by design, a lower drop probability threshold than the serving flows. If during probing, the new flow drop rate increases beyond a certain threshold, the flow is backpressured on the way to the source node and the flow is re-routed. If backpressure pushes the flow back to the source and all alternate paths are exhausted, the received flow is rejected. ProbeCast works well in small network density; however, the scalability in terms of network density and mobility is not guaranteed and the interference issue is not considered.

In [21], Pagani and Rossi proposed an end-to-end probing call multicast admission scheme, named MCAMP. In MCAMP, a source node, before transmitting data stream, floods probing packets to check the bandwidth availability along a multicast tree. Only the receivers take part in the admission control decision by sending an accept/refuse notification to the source based on the received quality. Three priority levels among packets are used in MCAMP: real time, probe, and best effort. The level 2 (probing packets) does not affect existing QoS flows. To deal with the mobility issue, a new bandwidth probing process is launched to re-construct the path and the allocation. However, in such implicit resource reservation model, the number of flows into

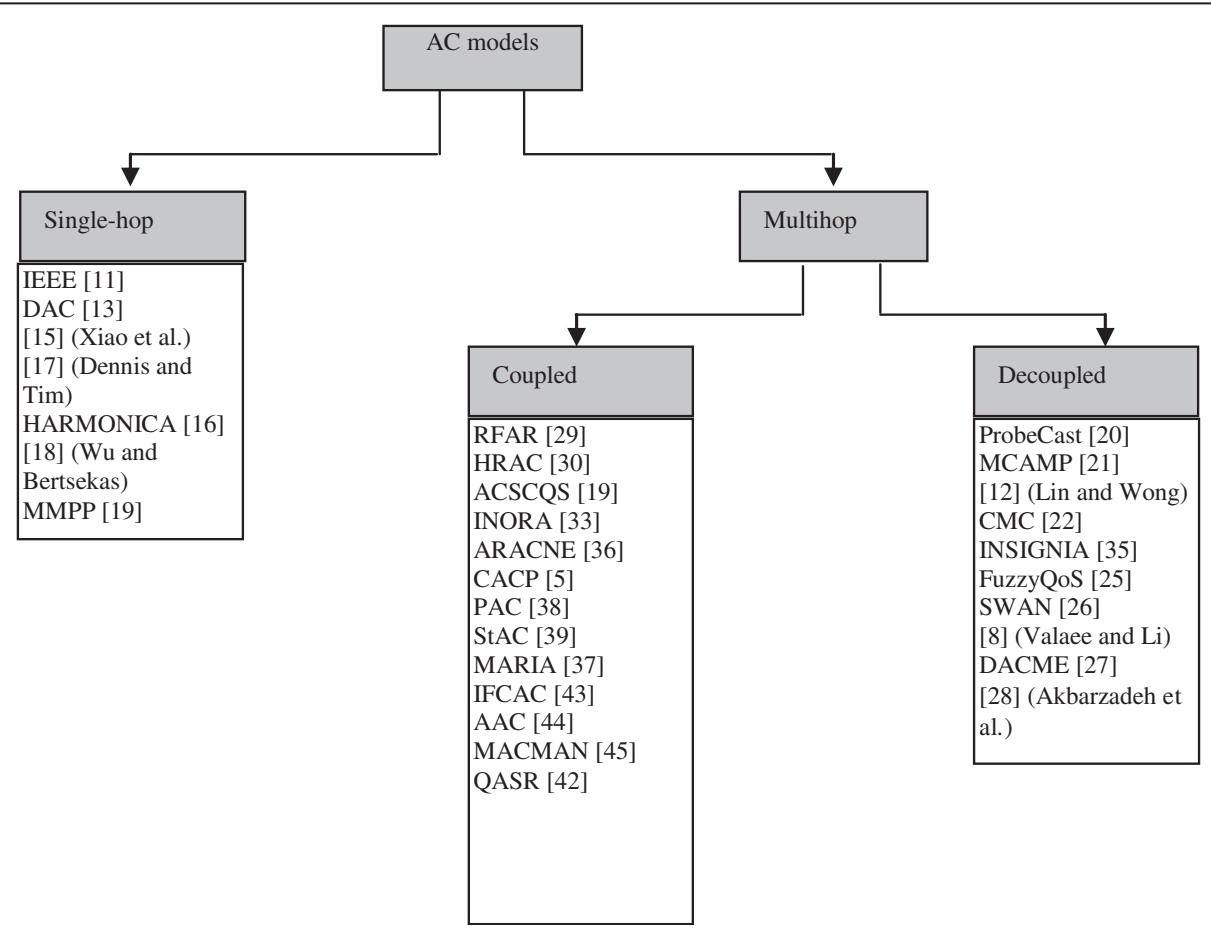

Figure 3 Coupled vs. decoupled routing AC model. 
the network is restricted to those that can achieve the target QoS.

In [12], Lin and Wong proposed a centralized admission control mechanism model based on the theory of conflict graph. The authors used a contention graph to model the contention situation in a multihop network, and they presented an analytical model to estimate the capacity for each maximal clique in the contention graph. A new session is admitted when the aggregated traffic load is less than the estimated network capacity. The model works well in a multihop single channel for a small-sized network. However, its main drawback is that the utilization of the conflict graph is highly complex; even for a moderate-sized network, the number of interference constraints can be very big.

Liu et al. [22] proposed a call admission control (CMC) model based on IEEE 802.11 multiradio multirate multichannel wireless mesh networks. CMC relies on local information to estimate the residual bandwidth of a path and can be integrated into existing routing protocols. The authors argued that CMC can correctly predict the end-to-end residual bandwidths of paths, successfully protects existing flows from QoS violations, and fully utilizes the bandwidth on channels. The contribution developed in [23] is based on delay parameter instead of throughput. It uses regression equations in the calculation of transmission probability which varies with each scenario. The authors made an important observation that an admission control algorithm that employs delay predictions as a threshold for call admission achieves, in theory, better channel utilization than those based on throughput parameter [24]. However, the work considers only a small network (four nodes are assumed to be actively transmitting flows).

In [25], the authors proposed FuzzyQoS, a stateless cross-layer AC protocol based on fuzzy logic theory for wireless ad hoc networks. The fuzzy approach aims to improve the control of traffic regulation rate and congestion control of multimedia applications. FuzzyQoS uses fuzzy thresholds to adapt the traffic transmission rate to the dynamic conditions. By monitoring the rate of change in queue length (variation rate) in addition to the queue length, FuzzyQoS provides a measure of queue state. Furthermore, by using explicit rate congestion notification, FuzzyQoS can make source nodes more responsive to sudden changes in the network traffic volume. The performance evaluation has shown that FuzzyQoS can achieve stable end-to-end delay under different network conditions. However, FuzzyQoS does not deal well with route failures. While searching for a new route, it reduces the data rate of affected sessions. This implies that FuzzyQoS can only support real-time applications with elastic throughput requirements.
In [8], Valaee and Li proposed a distributed call admission controller using a service-curve provisioning method, which reflects the status of network and depends on the number of active nodes, their activity index, and the back-off procedure used for contention resolution. The approach uses a sequence of small-sized probing packets to estimate the service curve of the network. Then, the estimated service curve is used to devise a call admission controller. As cited in [8], all users willing to start a new session should compare the performance of network to a non-decreasing deterministic function called the universal service curve. The authors assumed that the session requests are granted if the service curve stays above the universal service curve. Even the approach expresses a good performance under a small-sized network, the performance under high traffic load was not studied. Furthermore, the mobility factor was not considered in the approach.

In [26], the authors proposed a stateless service differentiation AC model, named SWAN. SWAN uses senderbased admission control in order to perform real-time traffic control. SWAN distinguishes between two traffic classes: real-time and best-effort, and it cooperates with almost all routing protocols. When a source station wants to send a real-time traffic to another station, it probes the path to the destination station to identify the bandwidth available for real-time traffic. SWAN relies on feedback information received from the MAC layer as a measure of congestion in the network by using mechanisms of rate control and source-based admission control. The AIMD rate control algorithm is used at each node in order to perform the control of best-effort traffic. The rate control restricts the bandwidth usage of best-effort traffic so that real-time applications can exploit the required bandwidth; the bandwidth not used by real-time applications can be exploited by the best-effort traffic. One limit of SWAN is that probing may cause a lot of overhead and packet loss.

In [27], Calafate et al. proposed a distributed admission control for MANET environments (DACME) model that handles multiconstrained real-time flows by periodically assessing end-to-end conditions on the path. In DACME, the source node performs path probing to obtain different QoS measurements of the path, thus assuring that the transmission of traffic is achieved under good conditions. The destination responds to probes, thus giving the source feedback about path conditions, avoiding sending information over paths possibly unavailable. DACME takes advantage of the IEEE 802.11e standard [7] to provide prioritized medium access. Even the model performs well in small to medium network, it suffers from fairness issue under high network load.

In [28], the authors investigated distributed algorithms for joint admission control, rate, and power allocation aiming at maximizing the flow's throughput. The 
admission decision is based on statistical knowledge of the channel and buffer states of communication pairs and on the exact knowledge of their own channel and buffer states. The authors also studied the benefits of a cross-layer approach compared to a conventional resource allocation ignoring the states of the queues. Even the proposed work was designed for large interference systems, the performance evaluation was not studied under a large number of active sessions. In addition to the scalability issue, the model did not consider the impact of mobility.

Routing-coupled AC schemes This sub-section summarizes some routing-coupled AC schemes which require that all intermediate nodes have routing capabilities to achieve admission decisions.

Zhang and Rubin [29] proposed a robust flow admission and routing (RFAR) protocol which incorporates new route robustness metric. RFAR aims to maximize the network's 'robust throughput' which depends on the idea that more credit should be given when a session is completed without interruption, i.e., without violating its QoS requirements for its entire intended duration. In RFAR, for each class of data, a threshold is set as the maximum tolerable probability that the route breaks before the requesting session ends. If, during the route discovery phase, the cumulative robustness of the partially discovered route indicates a route failure probability surpassing this threshold, then the route request is not forwarded. The main drawback of RFAR is that it relies on nodes being able to estimate their own speed, via GPS receivers or some location-determination system, and this may limit the application of RFAR.

In [30], Dong et al. proposed a hierarchical routingbased admission control (HRAC) protocol. In HRAC, a logical super-device network is established via periodic HELLO message broadcasts. This structure is an approximation of the dominating set notion, such that each mobile device is at most one hop away from a superdevice. The HELLO messages also distribute device channel utilization information. Each mobile device estimates its available capacity in a simple manner by dividing the raw channel capacity by the MAC overhead parameter (estimated through simulations); it then subtracts the total channel utilization of its neighbors. The main weakness of HRAC is that it does not consider the intra-route contention when calculating a session's capacity requirement.

The authors in [31] proposed an admission control and simple class-based QoS system (ACSCQS) which incorporates some simple extensions to QoS-ad hoc on-demand distance vector (AODV) [32]. As in QoSAODV, when searching for a constrained route for a new arrival flow, the route request carries the session's throughput requirement. Once the new session is admitted, each intermediate node monitors the rate at which it is receiving the session's data. If this is less than the session's specified minimum throughput requirement, a route error message is sent to the source, which must find a new route. ACSCQS also periodically verifies that the session's end-to-end delay requirement is being upheld. The performance evaluation has shown that ACSCQS provides some improvements over the AODV protocol. However, the method of establishing a node's available capacity was not specified, and the admission control strategy was very simplistic.

The authors in [33] proposed INORA (admission control employing in-band signaling and the temporally ordered routing algorithm) which is the combination of TORA [34] and INSIGNIA [35] protocols. In INORA, routing information, modeled as an acyclic-directed graph rooted at the destination node, are assumed to have already been discovered by TORA. When a flow request arrives, the data packets are automatically admitted and the INSIGNIA component attempts to set up soft-state reservations. The data packets follow a directed graph set up by TORA. If an intermediate mobile device detects that it has insufficient available resources (e.g., by comparison to the channel idle time ratio (CITR)) or its queue is full beyond a certain threshold level, it notifies the previous device on the path. This device then attempts to route the session via different downstream devices. If all of the intermediate nodes' resources are sufficient to support at least the session's minimum required throughput, reservations are set up along the path, as in INSIGNIA. The merit of INORA is that multiple paths can cooperatively support the session; nevertheless, this is only under a simplified interference model.

The authors in [36] proposed an ARACNE protocol which is an ant-based routing algorithm with $\mathrm{AC}$ and noise route selection (NE) mechanisms. The AC and NE mechanisms aim to deal with congestion problem and shortcut problem, respectively. The AC mechanism detects the congestion of a route by estimating the delay and load information during route discovery and thereby avoids utilizing those congested routes, while the $\mathrm{NE}$ mechanism introduces additive noise into route selection for discovering shortcut routes and thus improves route convergence. However, the work was tested only under low mobility and traffic in non-interference network.

A contention-aware admission control (CACP) model is proposed by Yang and Kravets [5]. This work provides admission control decision for flows in a single- and multiple-channel ad hoc network based on knowledge of both local resources at a node and the effect of admitting the new flow on neighboring nodes. CACP introduced a c-neighbor concept (nodes in carrier-sensing 
range) to characterize contention in wireless networks. Information about c-neighbors is obtained through multihop querying packets or querying packets sent with increased transmission power. A node makes admission decision based on its c-neighbor available bandwidth which is the smallest local available bandwidth of all of its c-neighbors. In CACP, the on-demand querying packets are crucial to effective admission control. The loss of these packets may lead to inaccurate and unreliable admission decisions [37].

In [38], the authors proposed an AC mechanism which operates like CACP model, named perceptive admission control (PAC). PAC uses passive monitoring to estimate the available capacity at the current node and its neighbors. It addresses the admission control problem by monitoring the wireless channel using channel busy time and dynamically adapting admission control decisions to enable high network utilization while preventing congestion. This mechanism has the advantage that it can be used with any QoS-aware strategy. Furthermore, in the case of mobility causing imminent congestion, the source nodes of affected sessions attempt to pause traffic transmission for a random back-off period. However, this protocol does not consider intra-flow interferences when making admission decisions.

Hanzo and Tafazolli [39] proposed a staggered admission control protocol (StAC) based on passive monitoring of the admission control protocol. StAC ensures that performance requirements of a new session are maintained in a multihop ad hoc network, where mobile devices check their local resources through CITR mechanism $[3,40]$. StAC is partially related to DSR [41], using its basic routing functionality. StAC strategy can be implemented using service that starts transmitting traffic with a low rate and then gradually increasing it until it achieves the required flow rate of the session. StAC strategy re-routes the session when a path failure occurs due to congestion or mobility, and it reserves some capacity for unseen interference [40].

Cheng et al. [37] proposed a mesh admission control and QoS routing with interference awareness (MARIA) to investigate the QoS support of real-time multimedia applications. MARIA uses the conflict graph theory to capture both inter- and intra-flow interferences. The available residual bandwidth is computed based on the maximal clique constraints in its local conflict graph to make distributed hop-by-hop admission control decision. Nodes exchange their flow information periodically and compute their available residual bandwidth based on the local maximal clique constraints. Admission decision is made based on the residual bandwidth at each node. However, the authors assumed a distance-based model with fixed channel capacity; this means that MARIA should integrate a measurement method which accommodates varying channel capacity and captures interference more accurately.

Chauhan and Nandi [42] proposed a QoS-aware stable path routing scheme, named QASR, which finds out routes that satisfy delay and bandwidth constraints based on signal stability. QASR uses signal stability along with QoS parameters as route selection criterion. The estimation of signal stability is achieved with the help of both signal strength and link stability. The bandwidth reservation is activated for the flow only when the real data flow arrives at the registered nodes. Nodes in QASR periodically share location and flow state information with their neighbors. Even QASR considers the mobility as a main parameter in the admission control policy, the scalability of the model, in terms of both traffic load and nodes mobility, was not studied. Furthermore, QASR depends on known location information to determine the distance between nodes in the network.

In [43], the authors proposed an interference-based fair call AC protocol (IFCAC). In IFCAC, as opposed to previously discussed protocols, the channel is not considered busy just because the sensed interference power exceeds the carrier-sensing threshold (cs-thresh). Each node allocates an equal amount of channel capacity to each of the transmitters in its cs-range. For each case of the possible relative interference source positions, IFCAC determines the capacity to allocate to each transmitter within the cs-range in the most appropriate way. However, the drawback of IFCAC is that the sessions requiring more than their fair share will not be admitted, or will have to decrease their transmission rate when new sessions arrive.

Cano et al. [44] proposed an adaptive admission control (AAC) which is an AC model that deals with many issues regarding QoS provisioning in MANET. The $\mathrm{AC}$ procedure in AAC is coupled with QoS-AODV-style route discovery. AAC provides accurate low-cost signaling technique to retrieve CS nodes' available bandwidth and includes a contention count calculation algorithm which adapts to the path's roughness. AAC defines the usable bandwidth as the smallest available bandwidth on the sensing range of a node. HELLO messages used to spread the bandwidth information are transmitted to only one hop containing the sender's bandwidth information and its one hop neighbor. AAC works better in moderate traffic load and mobility environment, and gives accuracy information about the residual/required capacity estimation at nodes; however, this is only shown for a small-scale network.

Lindgren and Belding-Royer [45] proposed a multipath admission control for mobile ad hoc networks (MACMAN) which offers multiple paths/routes for the same data flow and thus improves the QoS. The source node selects the best route on some specified criteria and 
transmits the flow. The basic functionality of MACMAN is similar to CACP [5] and PAC [38]. The local residual capacity at nodes is tested in a manner similar to the PAC model, while the intra-route contention is taken into account in a way similar to CACP. One merit of PAC is that at any time, the backup paths (i.e., paths which satisfy a session's throughput requirement) are known by the traffic sources. This is ensured by the fact that each backup path is regularly tested to have adequate end-to-end capacity for the accepted session. Nevertheless, this testing process may generate an additional overhead.

\section{Conclusions}

The admission control decision in MANETs is typically based on some predefined criteria, which depends on the network traffic state and the characteristics of incoming sessions (e.g., their required network bandwidth). The design of AC models poses several challenges as described in the 'Design challenges of AC models in MANETs' section. These challenges are potential sources of service impairment in ad hoc networks, and hence, they may degrade the QoS seen by users of the network. An AC model should guarantee a successful data delivery with a good quality under a given limited memory, buffer and processing capabilities of mobile devices, implemented algorithm complexity, and wireless environment constraints. The overhead-heavy AC models may have a significant impact on resource and battery life and hence may limit the usefulness of devices. A detailed analysis of the previous discussed AC schemes is discussed in what follows.

\section{AC contribution analysis}

We have summarized the classification of the AC models described in the 'AC models in ad hoc networks' section in Tables 1 and 2; hereafter, we review their major features.

\section{Single-hop vs. multihop MANET}

In practice, designing $\mathrm{AC}$ models for multihop networks poses more challenges than for single-hop networks because of the fact that there is no centralized control in multihop networks (i.e., MANETs). Thus, any multihop AC strategy should support the distributed nature of ad hoc networks. The majority of single-hop AC models existing in the literature (e.g., DAC, MMPP, [15,17]) are proposed to improve the IEEE 802.11e. In a single-hop 802.11e, the traffic differentiation works well in small to medium network load. However, when the traffic load becomes high, increasing contention may lead to excess retransmissions and collisions, which leads to an increase of delay and a decrease in network throughput. Our general remark is that almost single-hop AC models (e.g., [7,13-15]) require complete network topology information at the central point (QAP); it is clear that such schemes can only protect existing flows when the traffic load is not heavy; thus, the scalability is not always guaranteed. One manner to deal with this issue is by expecting the achievable throughput of incoming sessions and avoiding channel overloading, as proposed in [17]. We think also that the scalability can be improved by applying a 'soft admission control', which allows the possibility of adapting the rate of existing sessions according to both dynamic network conditions and acceptable threshold user requirements. By adapting the rate of existing flows, more bandwidth will be available in network; this leads to 'facilitate' the admission process of new sessions. Indeed, the priority of traffic should be the important parameter to take into consideration for both the rate adaptation of existing traffic and the admission of new flows.

\section{Coupled vs. decoupled AC models}

As our detailed survey showed, some AC models (e.g., [12], CMC, IFCAC, StAC, DACME, [28]) are decoupled from routing schemes; they suppose that a route for a requesting flow has already been discovered, and their main task is to evaluate the route's fitness for supporting the flow's requirements. Thus, they are totally free from the routing scheme deployed in the network.

The main advantage of these decoupled models is that they are able to operate in conjunction with any routing protocol. A number of decoupled models are stateless AC models (e.g., SWAN, FuzzyQoS), which aim to offer $\mathrm{AC}$ decision without requiring prior resource reservation and thus overcoming the overhead restrictions of $\mathrm{AC}$ state models. In such stateless models, the traffic differentiation is realized according to the class they belong to without maintaining any state information. Decoupling the admission control function from the routing protocol may save the often broadcast-natured overhead that is typically encountered in protocols testing the resources of neighboring nodes (Figure 4). However, one of the drawbacks of this kind of models is that the paths obtained by the discovery route process may not be always useful to any sessions (due to a scarcity of route resources).

To overcome this problem, the idea of coupling AC models with a routing scheme may be interesting, such as implemented in CACP, PAC, INORA, AAC, etc. The time required to achieve the process of admission control in such models is smaller than that required in decoupled AC models because the latter should wait for a separate route discovery process to be achieved by an implemented routing protocol. Even the abundance of information in such approaches leads to accurate admission decisions (Figure 4), the performance of the coupled AC models may depend on the efficiency of the used stateful routing scheme. For instance, 
Table 1 Comparison of AC models

\begin{tabular}{|c|c|c|c|c|c|c|c|c|c|c|c|c|c|}
\hline \multirow[t]{2}{*}{ Protocol/algorithm } & \multicolumn{2}{|c|}{ State category } & \multicolumn{3}{|c|}{$\begin{array}{l}\text { Interaction } \\
\text { routing/QoS }\end{array}$} & \multicolumn{2}{|c|}{$\begin{array}{l}\text { Wireless } \\
\text { connection }\end{array}$} & \multicolumn{3}{|c|}{ Model layer } & \multicolumn{3}{|c|}{$\begin{array}{l}\text { Performance constraint } \\
\text { consideration }\end{array}$} \\
\hline & STAT & STLS & $\mathrm{CP}$ & DP & $\mathrm{HY}$ & SH & $\mathrm{MH}$ & Net & MAC & $\mathrm{CL}$ & TH & D & JP \\
\hline DAC [13] & & $x$ & & & $x$ & $x$ & & & $x$ & & $x$ & $x$ & $x$ \\
\hline INORA [33] & $x$ & & $x$ & & & & $x$ & & & $x$ & $x$ & & \\
\hline IFCAC [43] & & $x$ & & $x$ & & & $x$ & & $x$ & & & $x$ & $x$ \\
\hline SWAN [26] & & $x$ & & $x$ & & & $x$ & & & $x$ & $x$ & $x$ & \\
\hline HARMONICA [16] & & $x$ & & & $x$ & $x$ & & & $x$ & & $x$ & $x$ & \\
\hline ACSCQS [19] & & $x$ & $x$ & & & & $x$ & $x$ & & & $x$ & $x$ & $x$ \\
\hline RFAR [29] & $x$ & & $x$ & & & & $x$ & $x$ & & & $x$ & $x$ & \\
\hline PAC [38] & & $x$ & & $x$ & & & $x$ & & $x$ & & $x$ & $x$ & \\
\hline HRAC [30] & $x$ & & $x$ & & & & $x$ & $x$ & & & $x$ & & \\
\hline [15] (Xiao et al.) & $x$ & & & & $x$ & $x$ & & & $x$ & & $x$ & $x$ & $x$ \\
\hline StAC [39] & & $x$ & & & $x$ & & $x$ & & & $X$ & & $x$ & $x$ \\
\hline MARIA [37] & $x$ & & & $x$ & & & $x$ & & $x$ & & $x$ & $x$ & X \\
\hline CACP [5] & $x$ & & $x$ & & & & $x$ & & & $X$ & $x$ & & \\
\hline AAC [44] & $x$ & & $x$ & & & & $x$ & $x$ & & & $x$ & & \\
\hline FuzzyQoS [25] & & $x$ & & $x$ & & & $x$ & & & $x$ & & $x$ & $x$ \\
\hline MACMAN [45] & $x$ & & $x$ & & & & $x$ & & & $x$ & $x$ & & $x$ \\
\hline MCAMP [21] & & $x$ & & $x$ & & & $x$ & & & $x$ & $x$ & & \\
\hline [23] (Bai et al.) & & $x$ & & & $x$ & & $x$ & & & $x$ & & $x$ & \\
\hline ARACNE [36] & $x$ & & $x$ & & $x$ & & $x$ & $x$ & & & $x$ & $x$ & \\
\hline [12] (Lin and Wong) & $x$ & & & $x$ & & $x$ & $X$ & & $x$ & & $x$ & $x$ & \\
\hline [18] (Wu and Bertsekas) & $x$ & & & $x$ & & $x$ & & & $x$ & & & & $x$ \\
\hline ProbeCast [20] & & $x$ & & & $x$ & & $x$ & & & $x$ & $x$ & & $x$ \\
\hline [17] (Dennis and Tim) & $x$ & & & $x$ & & $x$ & & & $x$ & & $x$ & $x$ & \\
\hline QASR [42] & $x$ & & $x$ & & & & $x$ & $x$ & & & $x$ & $x$ & $x$ \\
\hline [8] (Valaee and Li) & & $x$ & & $x$ & & & $x$ & & $x$ & & & $x$ & $x$ \\
\hline DACME [27] & & $x$ & & $x$ & & & $x$ & & & $x$ & $x$ & $x$ & $x$ \\
\hline [28] (Akbarzadeh et al.) & $x$ & & & & $x$ & & $x$ & & & $x$ & $x$ & & $x$ \\
\hline MMPP [19] & & $x$ & & $x$ & & $x$ & & & $x$ & & & $x$ & \\
\hline
\end{tabular}

STAT, stateful; CP, coupled; SH, single-hop; Net, network layer; TH, throughput; STLS, stateless; DP, decoupled; MH, multihop; MAC, MAC layer; D, delay; HY, hybrid; $\mathrm{CL}$, cross-layer; JP, jitter/packet loss, etc.

some proactive routing schemes (e.g., OSPF-MCDS [46]) require an extensive message exchange, which may produce an additional overhead in the network and consequently may impact negatively on the admission control decision (Figure 4). In addition, some
AC source-based coupled models (e.g., MACMAN, CACP, ACSCQS) are relied on outdated information (especially in high-dynamic MANETs), which may incur a burden of local available bandwidth estimation at intermediate nodes.

Table 2 Features of AC approaches

\begin{tabular}{|c|c|c|c|c|c|c|}
\hline & & $\begin{array}{c}\text { MAC technology } \\
\text { operability }\end{array}$ & $\begin{array}{c}\text { Communication } \\
\text { overhead }\end{array}$ & $\begin{array}{l}\text { Sensitivity } \\
\text { to mobility }\end{array}$ & $\begin{array}{l}\text { Sensitivity to false } \\
\text { admission issue }\end{array}$ & Scalability \\
\hline \multirow[t]{2}{*}{ Routing-coupled } & MAC-dependent & No & High & High & Lightweight & Not scalable \\
\hline & MAC-independent & Yes & High & High & Lightweight & Not scalable \\
\hline \multirow[t]{2}{*}{ Routing-decoupled } & MAC-dependent & No & Moderate & Moderate & High & Scalable \\
\hline & MAC-independent & Yes & Lightweight & Lightweight & High & Scalable \\
\hline
\end{tabular}




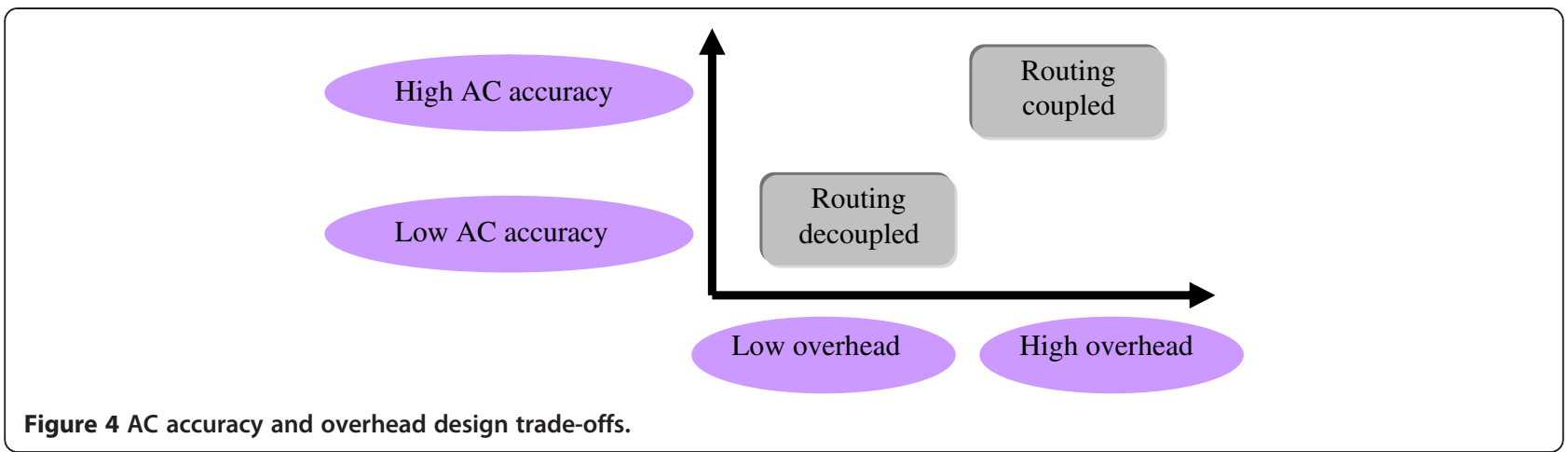

\section{Implicit vs. explicit reservation AC models}

To achieve the AC decision in both coupled and decoupled models, resources must be reserved, either implicitly or explicitly. In the first mode, as described previously, there are no resources associated with a particular session. When a new session is to be admitted into the network, an end-to-end probing is used to determine whether the flow requirement (e.g., bandwidth) is acceptable. Thus, the network is viewed as a black box, and the number of sessions into the network is restricted to those that can achieve the network QoS availability. The implicit mode is applied in some stateless AC models (e.g., FuzzyQoS, MCAMP, DACME, SWAN, PAC). The advantage of such mode is that it offers the scalability since no session state information is maintained at intermediate nodes (Table 2). On the other side, almost coupled AC models use the explicit reservation mode, where each node associates resources to a particular flow. These models have been carried out under the concept of per-flow granularity, so the amount of state information increases proportionally with the number of flows. This may result in a processing and storage overhead on nodes.

\section{MAC-coupled vs. MAC-decoupled AC models}

An AC model is MAC-coupled if it uses information directly given by the MAC layer for estimating the residual channel capacity. Some previous described works (e.g., DACME) assumed an EDCA MAC scheme, but they can also operate with a non-QoS-aware MAC like the basic 802.11 distributed coordination function (DCF). In MANETs, as observed in [9], contention-free MAC schemes such as code division multiple access or time division multiple access are complex to implement due to the dynamically changing network topology and the lack of centralized control.

Note that almost all earlier routing-coupled and routing-decoupled AC models are based on DCF, for example, IFCAC, PAC, HRAC, StAC, CACP, ACSCQS, MACMAN, AAC, etc. On the other hand, the AC models which are decoupled from the MAC layer (e.g., RFAR,
SWAN, FuzzyQoS, [12,28]) can utilize only information of the network layer; this leads to a simpler interaction with different MAC technologies without need to a complex cross-layer design, but at the expense of false admission issue (Table 2).

\section{Interference-aware concern}

Because the interference among concurrent transmissions may complicate the QoS support in wireless networks, some models, such as MARIA and CACP, have given a special attention to this issue. When a mobile node admits a session, the interferences from existing session's flows in its transmission range should be considered because the acceptance of a new arrival flow can affect the existing flows. While some models, like INORA, allow multiple routes to cooperatively support the session but only under a simplified interference cases, other models (e.g., MARIA, CACP) have implemented a routing scheme that explicitly incorporates an interference model based on local maximal clique constraints in the route discovery process. Some models (e.g., [12,47]) dealing with the interference issue use the conflict graph which is highly complex; even for a small network, the number of interference constraints can be very big. Note also that some earlier works (e.g., HRAC, DACME, INSIGNIA, INORA) ignored the impact of intra-route contention on a flow's capacity requirement prior to admission decision. These AC works mistreated any effect that accepting the flow would have on mobile nodes which are not on the flow's route.

\section{Mobility-aware concern}

The mobile devices in an ad hoc network may move randomly; a transmitting node may move into the sensing range of another transmitter, thus increasing its interference. Some works (e.g., CACP) do not propose any strategy to handle mobility and loss of QoS guarantees. The survey has revealed that there are only a few models, like QASR, StAC, DACME, MACMAN, RFAR, that make a serious attention to consider mobility as the main factor in the design of AC models. In order to deal 
with the QoS violation caused by the mobility impact, some other works (e.g., $[30,31,33,43]$ ) rely on the deployed routing mechanism to re-route affected flows and modify the $\mathrm{AC}$ decision each time a flow is rerouted. While most stateful AC models at least consider this possibility, almost stateless AC models (e.g., SWAN, ProbeCast, FuzzyQoS) may suffer from the false admission issue (Table 2) due partially to the effect of mobility, and this is caused by the fact that each source node may receive a notification message (from mobile intermediate or destination nodes) indicating that resources are available in the network, when in fact they might not.

\section{Summary and future research directions}

In this survey, we presented a review of the literature on the admission control concept in single- and multihop networks. Several design issues concerning the development of an AC model for ad hoc networks have been discussed. Then, a detailed investigation of current research on AC models for ad hoc networks has been achieved.

In line with the ultimate aims of current $\mathrm{AC}$ research, the goals of future work on admission control strategies in MANET are twofold: firstly, to make the design of AC models more 'intelligent' by combining several AC schemes. Thus, two or more AC schemes can be used for different applications (interference-sensitive, delaysensitive, etc.) or different network sizes. For instance, ProbeCast can be used efficiently for a small to medium network size, but when the network becomes large (as a large number of mobile nodes joint the network), SWAN may be activated to ensure the traffic admission decision. Indeed, an efficient use of resources in such concept should be carefully considered. Secondly, it is to make the initial resource reservation process (used, for instance, in coupled AC models) more flexible and 'lighter,' leading to reduced amount of information stored at intermediate nodes. For that aim, we believe that intelligent hybrid AC models that combine the advantages of both stateful and stateless AC models and adapt the traffic rate to the state of the network resources would be of practical significance. The ideal plan for future AC strategies would be able to predict the changes in MANET's topology and the availability of resources before they happen and then to run the admission decision according to the estimated resources (e.g., buffer level, power level, etc.) in the network. We believe that the performance investigation of $\mathrm{AC}$ models supporting high MANET mobility in other dynamic networks (e.g., vehicular networks) would be very useful.

The advantage and the cost of a particular AC strategy must be seen in close conjunction with antenna-based technologies. The use of multiple antennas or directional antenna-based systems would be very interesting to alleviate the capacity limits. However, such technologies have also some drawbacks, like difficulty in preserving the exact transmission direction in the face of mobility and higher directional interference, in the case of directional antenna systems. We believe that the design of AC strategies, for low-mobility applications, using directional antenna systems would be an attractive solution for future QoS wireless communication.

\section{Competing interests}

The authors declare that they have no competing interests.

\section{Acknowledgements}

This research was supported in part by the University of Technology of Troyes (UTT-France) under grant ER-210 2010-2013.

\section{Author details}

'Autonomic Networking Environment, CNRS UMR-STMR 6279, University of Technology of Troyes, Troyes 10000, France. ${ }^{2}$ Computer Sciences Department, University of Marne la Vallée, Paris 77420, France.

Received: 1 April 2012 Accepted: 25 March 2013

Published: 23 April 2013

\section{References}

1. S Chakrabarti, A Mishra, QoS issues in ad hoc wireless networks. IEEE Commun. Mag. 39(2), 142-148 (2001)

2. P Gupta, PR Kumar, The capacity of wireless networks. IEEE Trans. Inf. Theory 46(2), 388-404 (2000)

3. IEEE Computer Society, IEEE Standard 802.11-2007: Wireless LAN Medium Access Control (MAC) and Physical Layer (PHY) Specifications (IEEE, Piscataway, 2007)

4. H Lajos, T Rahim, Admission control schemes for 802.11-based multi-hop mobile ad hoc networks: a survey. IEEE Communications Surveys \& Tutorials 11(4), 78-108 (2009)

5. Y Yang, R Kravets, Contention-aware admission control for ad hoc networks. IEEE Trans. Mob. Comput. 4, 363-377 (2005)

6. X Xiang, X Wang, Y Yang, Stateless multicasting in mobile ad hoc networks. IEEE Trans. Comput. 59(8), 1076-1090 (2010)

7. B Zhang, HT Mouftah, QOS Routing for wireless ad hoc networks: problems, algorithms, and protocols. IEEE Commun. Mag. 43(10), 110-115 (2005)

8. S Valaee, B Li, Distributed call admission control for ad hoc networks, in Proceedings of the IEEE Vehicular Technology Conference (VTC) (Vancouver, 24-28 Sep 2002)

9. L Hanzo, R Tafazolli, A survey of QoS routing solutions for mobile ad hoc networks. IEEE Communications Surveys \& Tutorials 9(2), 50-70 (2007)

10. S Saunders, Antennas and Propagation for Wireless Communication Systems: Concept and Design (Wiley, New York, 1999)

11. IEEE 802.11 WG, IEEE Std 802.11e-2005 (Amendment to IEEE Std 802.11, 1999 Edition (Reaff 2003) (IEEE, Piscataway, 2005)

12. $Y$ Lin, WWS Wong, An admission control algorithm for multi-hop 802.11e based WLANs. Computer. Communication 31(14), 3510-3520 (2008)

13. Y Xiao, $\mathrm{H}$ Li, Voice and video transmissions with global data parameter control for the IEEE 802.11e enhance distributed channel access. IEEE Transactions on Parallel and Distributed Systems 15(11), 1041-1053 (2004)

14. D Gao, J Cai, K Ngan, Admission control in IEEE 802.11e wireless LANs. IEEE Networks 19(4), 6-13 (2005)

15. Y Xiao, H Li, S Choi, Protection and guarantee for voice and video traffic in IEEE 802.11e wireless LANs, in Proceedings of IEEE INFOCOM, vol. 3 (Hong Kong, 7-11 March 2004), pp. 2152-2162

16. L Zhang, S Zeadally, HARMONICA: enhanced QoS support with admission control for IEEE 802.11 contention-based access, in Proceedings of the IEEE Real-Time and Embedded Technology (Toronto, 25-28 May 2004), pp. 64-71

17. P Dennis, M Tim, Call admission control for IEEE 802.11 contention access mechanism, in Proceedings of the IEEE GlobeCom (San Francisco, 1-5 Dec 2003)

18. CC Wu, DP Bertsekas, Admission control for wireless networks. IEEE Trans. Veh. Technol. 50, 504-514 (2001) 
19. A Abdrabou, W Zhuang, Stochastic delay guarantees and statistical call admission control for IEEE 802.11 single hop ad hoc networks. IEEE Trans. Wirel. Commun. 7(10), 3972-3981 (2008)

20. SY Oh, G Marfia, M Gerla, MANET QoS support without reservations. Journal of Security and Communication Networks 4(3), 316-328 (2011)

21. E Pagani, GP Rossi, A framework for the admission control of QoS multicas traffic in mobile ad hoc networks, in Proceedings of the ACM International Workshop on Wireless Mobile Multimedia (ACM, New York, 2001), pp. 2-11

22. T Liu, W Liao, J Lee, Distributed contention-aware call admission control for IEEE 802.11 multi-radio multi-rate multi-channel wireless mesh networks. Journal of Mobile Networks and Applications 14(2), 134-142 (2009)

23. A Bai, T Skeie, PE Engelstad, A model-based admission control for 802.11e EDCA using delay predictions, in Proceedings of the IPCCC (Phoenix, 11-13 Apr 2007)

24. K Anna, M Bassiouni, A distributed admission control to lower end-to-end delay in p-persistent 802.11 MAC protocol, in Proceedings of the IEEE Wireless Telecommunications Symposium (WTS) (Pomona, 24-26 Apr 2008), pp. 316-323

25. L Khoukhi, S Cherkaoui, Intelligent QoS management for multimedia services support in wireless mobile ad hoc networks. Computer Networks 54(10), 1692-1706 (2010)

26. GH Ahn, AT Campbell, A Veres, LH Sun, SWAN: Service differentiation in stateless wireless ad hoc networks, in Proceedings of the IEEE INFOCOM (New York, 23-27 June 2002), pp. 457-466

27. CT Calafate, MP Malumbres, J Oliver, JC Cano, P Manzoni, QoS support in MANETs: a modular architecture based on the IEEE 802.11e technology. IEEE Transactions on Circuits and Systems for Video Technology 19(5), 678-692 (2009)

28. S Akbarzadeh, L Cottatellucci, C Bonnet, Low complexity cross-layer design for dense interference networks, in Proceedings of the International Symposium on Modeling and Optimization in Mobile, Ad-Hoc and Wireless Networks (WiOpt) (Seoul, 23-27 June 2009)

29. R Zhang, I Rubin, Robust flow admission control and routing for mobile ad hoc networks, in Proceedings of the IEEE Military Communications (Washington, DC, 23-25 Oct 2006), pp. 1-7

30. Y Dong, D Makrakis, T Sullivan, Effective admission control in multihop mobile ad hoc networks, in Proceedings of the IEEE International Communication Technology, vol. 2 (Beijing, 9-11 Apr 2003), pp. 1291-1294

31. M Haq, M Matsumoto, J Bordim, M Kosuga, S Tanaka, Admission control and simple class-based QoS provisioning for mobile ad hoc network, in Proceedings of the IEEE 60th Vehicular Technology Conference (Los Angeles, 26-29 Sep 2004)

32. CE Perkins, EM Belding-Royer, Quality of service for ad hoc on-demand distance vector routing (Fremont, IETF Internet Draft, 2003)

33. D Dharmaraju, A Roy-Chowdhury, P Hovareshti, J Baras, INORA - a unified signaling and routing mechanism for QoS support in mobile ad hoc networks, in Proceedings of the IEEE International Conference on Parallel Processing Workshop (Vancouver, 18-21 Aug 2002), pp. 86-93

34. V Park, S Corson, Temporally Ordered Routing Algorithm v1 Functional Specification (IETF Internet Draft, Fremont, 1997)

35. S Lee, G Ahn, X Zhang, A Campbell, INSIGNIA: an IP-based quality of service framework for mobile ad hoc networks. Journal of Parallel and Distributed Computing 60(4), 374-406 (2000)

36. R Liu, W Guo, X Zheng, T Yan, On the congestion and shortcut problems of ant-based routing for mobile ad hoc networks, in Proceedings of the International Conference on Communications, Circuits and Systems (Hong Kong, 27-30 May 2005)

37. X Cheng, P Mohapatra, S-J Lee, S Banerjee, MARIA: interference-aware admission control and QoS routing in wireless mesh networks, in Proceedings of the IEEE International Conference on Communications (Beijing, 19-23 May 2008), pp. 2865-2870

38. ID Chakeres, EM Belding-Royer, PAC: perceptive admission control for mobile wireless networks, in Proceedings of the IEEE International Conference on Quality of Service in Heterogeneous Wired/Wireless Networks (Washington, DC, 18-20 Oct 2004), pp. 18-26

39. L Hanzo, R Tafazolli, Throughput assurances through admission control protocol for multi-hop MANETs, in Proceedings of the IEEE International symposium. Personal, Indoor and Mobile Radio Communication (Athens, 3-7 Sep 2007), pp. 1-5

40. A Khan, K Khattak, AC and QAR for provisioning of QoS in MANETS. Master thesis (Blekinge Institute of Technology, 2010)
41. D Johnson, D Maltz, Y Hu, The Dynamic Source Routing Protocol (DSR) for Mobile Ad-hoc Networks. IETF MANET Working Group Experimental RFC 4728 (IETF, Fremont, 2007)

42. G Chauhan, S Nandi, QoS aware stable path routing (QASR) protocol for MANETs, in Proceedings of the International Conference on Emerging Trends in Engineering and Technology (Nagpur, 16-18 July 2008), pp. 202-207

43. K Sridhar, M Chan, Interference-based call admission control for wireless ad hoc networks, in Proceedings of the International Conference on Mobile and Ubiquitous Systems: Networking \& Services (San Jose, 17-21 July 2006), pp. $1-10$

44. C Cano, B Bellalta, M Oliver, Adaptive admission control mechanism for IEEE 802.11E WLANS, in Proceedings of the IEEE International Symposium on Personal, Indoor and Mobile Radio Communication (Athens, 3-7 Sep 2007)

45. A Lindgren, EM Belding-Royer, Multi-path admission control for mobile ad-hoc networks, in Proceedings of the International ICST Conference on Mobile and Ubiquitous Systems: Networking and Services (San Diego, 17-21 July 2005), pp. 407-417

46. T Lin, SF Midkiff, JS Park, Approximation algorithms for minimal connected dominating sets and application with routing protocol in wireless ad hoc networks, in Proceedings of the IEEE International Performance Computing and Communications Conference (Phoenix, 9-11 Apr 2003), pp. 157-164

47. Q Junaid, CT Chou, A Misra, JG Lim, Minimum latency broadcasting in multiradio, multichannel, multirate wireless meshes. IEEE Trans. Mob. Comput. 8(11), 1510-1523 (2009)

doi:10.1186/1687-1499-2013-109

Cite this article as: Khoukhi et al: Admission control in wireless ad hoc networks: a survey. EURASIP Journal on Wireless Communications and Networking 2013 2013:109.

\section{Submit your manuscript to a SpringerOpen ${ }^{\odot}$ journal and benefit from:}

- Convenient online submission

- Rigorous peer review

- Immediate publication on acceptance

- Open access: articles freely available online

- High visibility within the field

- Retaining the copyright to your article

Submit your next manuscript at $\gg$ springeropen.com 